\title{
The Production and preservation technology of human anatomy specimens and its development
}

\author{
Shang Qi , Wei Dequan \\ Zaozhuang Vocational College of Science \& Technology, Tengzhou,Shandong, 277500,China
}

Keywords: Human anatomy specimens; Production and preservation technology; development; Plasticized specimens

\begin{abstract}
At present, with the continuous development of medical technology, the production and preservation of human anatomical specimens are continuously updated. Due to the traditional production and preservation of human anatomical specimens mainly follow the previous approach. While, the proper production and preservation of human anatomical specimens will directly affect the experimental teaching and research work. Based on this, this article uses the literature review method, case demonstration method to start from the overview of human anatomy specimens. Then, according to the current situation of the production and preservation of human anatomical specimens, the technology and development of production and preservation of human anatomy specimen are studied. The purpose is to provide some reference for the researchers in the production and preservation of human anatomical specimens and to indicate the development direction for the production of high quality human anatomical specimens in the future.
\end{abstract}

\section{Introduction}

With the emergence of new medical technologies and new methods, especially the development of technologies, such as ultrasound and CT have been widely used in clinical[1]. The human anatomy specimens, which occupies an important position in anatomy teaching. the medical profession has done a lot of research on the production and preservation of human anatomical specimens. Although a lot of experience and specific examples have been accumulated, there is still a certain gap compared with the technology of making and preserving human anatomical specimens abroad. Based on our own experience, we illustrate the current situation and development of preservation technology of human anatomy specimens, and make unremitting efforts to make high-level human anatomical specimens.

\section{An overview of human anatomic specimens}

Human specimens are made from the real human body after a series of chemical treatments. For human anatomical specimens, they are preserved after a series of chemical treatments in accordance with posture of human anatomy.They can elucidate the shape, location and interrelationships of various ministries and structures in the human body [2]. Table I is a specific human anatomy specimen production process In the field of education, human anatomy specimens as an important part of anatomy teaching can help students in the theory of true understanding and mastery of anatomy. Because traditional human anatomy specimens are preserved by formalin solution with pungent odor, it not only affects the physical and mental health of teachers and students, but also restricts the teaching effect. However, with the development of medical technology, the current education circles and relevant researchers have set new requirements on the production and preservation of human anatomical specimens. 
Table I human anatomy specimens making process

\begin{tabular}{|c|c|}
\hline steps & requirements \\
\hline $\begin{array}{c}\text { Material } \\
\text { selection }\end{array}$ & $\begin{array}{c}\text { The bodies are young adults of normal morphology, less subcutaneous } \\
\text { adipose tissue, no physiological defects and special diseases. }\end{array}$ \\
\hline $\begin{array}{c}\text { positioning } \\
\text { by marking }\end{array}$ & $\begin{array}{c}\text { According to the body surface sign, using the bomb method in the body } \\
\text { surface positioning and marking, the depth is 1-2cm }\end{array}$ \\
\hline $\begin{array}{c}\text { Dismember } \\
\text { the body }\end{array}$ & $\begin{array}{c}\text { Using the MJ-346B carpenter sawing machine, and it is necessary to } \\
\text { debug the instrument and test sawing before sawing. }\end{array}$ \\
\hline $\begin{array}{l}\text { Bulk } \\
\text { process the } \\
\text { specimen }\end{array}$ & $\begin{array}{c}\text { According to the specimen production requirements, removing } \\
\text { unnecessary tissue structure and maintaining the integrity of the tissue } \\
\text { structure when removed }\end{array}$ \\
\hline $\begin{array}{c}\text { Fine } \\
\text { processing }\end{array}$ & The specimens are treated with bleaching (10\% hydrogen peroxide), \\
soaking (12h) and sun drying.
\end{tabular}

\section{The technology and development of current human anatomy specimens production and preservation}

With the continuous development of modern science and technology, the production and preservation technology of human anatomical specimens are continuously updated.According to the relevant research, the development of human anatomy and preservation technology has entered a new stage so far. According to the current production and preservation of anatomical specimens of human anatomy, We explain the current technology and development of human anatomy specimen preservation through ordinary human specimens, human mummy specimens, human cast specimens and bio plasticized specimens. The following is a concrete content:

\section{A. Ordinary human specimens}

\section{a. Production}

The body is required to be fixed when the common specimens are made. The general operation is to cut through the skin on the body side of the lower part of the femoral triangle, blunt dissected out of the femoral artery, the femoral artery was wall slit, with T-tube. One end can be inserted into the blood vessel, and one end can be connected with the phase rubber tube. A bucket perfusion method was used to carry out an injection[3]. The general filling material of $8 \%$ of formaldehyde is good. To 17-50-year-old body, for example, the perfusion volume of 5000-12000ml. For the processing of the body of the broken cranial, the plastic wrap can be used on the head wrapped, it is best to tie the neck with fine iron wire to prevent liquid leakage.In the process of specimen making, we should do our best to conduct scientific design and production. According to the overall should be rough, local for fine principle, we can make a reasonable distinction and choice between the main and the subordinate, and there must be a long-term plan.

\section{B. Preservation}

For the preservation of ordinary human specimens, it is mainly drug preservative, vacuum corrosion, dry preservation and low temperature preservation. And different human specimens, the ways of preservation are different, and the following are explained in detail.

\section{a.The anatomical and anatomical autopsy specimens}

This type of specimens is preserved mainly by the preservative formalin solution pool.The general pool consists of six pools, equipped with three sets of ventilation devices, and the roof has an automatic track system. Each large pool contains 16 small pool, each pool holds a specimen, the pool has formaldehyde solution, alcohol and glycerol and a large amount of tap water. For example, a body that has been soaked in rainwater may be stored after the body has been treated for mildew and found to be mildew-free.

\section{b. Local anatomy and fragmented specimens}

Local specimens are mainly stored in large urn.The fragmented small single organ samples, such as liver, heart, intestine and brain samples, etc., can be placed in pots. Figure I is the whole brain specimen production. In the preservation process, this kind of specimens requires a concentration of 
$8 \%$ formaldehyde solution, while the brain tissue preservation at $10 \%$.The drug concentration can be checked for once every three months.

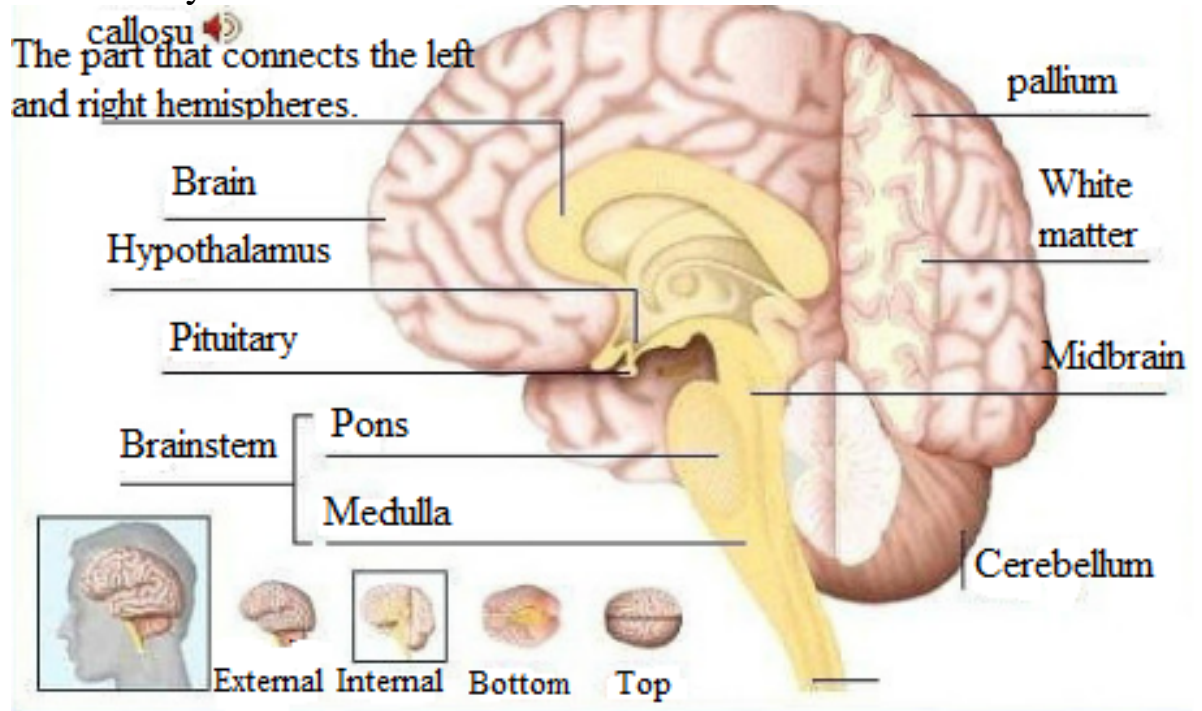

Figure I whole brain specimen production

\section{c.Human vascular nerve stained whole body specimens, department of solution specimens and tomographic specimens}

For this kind of specimen, organic glass cylinder or organic glass cabinet are used for preservation. In the preservation process, we should pay great attention to maintain a certain distance between small specimens and plexiglass cylinder edge. It can be controlled at $2 \mathrm{~cm}$ or more to prevent the specimen from being squeezed and deformed[4]. The distance between the large specimens and glass cylinder edge can be controlled at $4 \mathrm{~cm}$ above. For example, a specimen such as a general system overview needs to be placed flat on a bench so as to avoid collisions and scratches on the plexiglass cabinet, and a cover can be added on the outside of the glass cabinet.It is usually sealed by wet preservation. The chemical solution used is $5 \%$ formaldehyde and distilled water. The concentration of the solution could be checked again for 3 months.

\section{d.A whole body specimen for experimental teaching}

The blood vessel and nerve must be kept intact and the superficial muscles should be displayed clearly when producing such specimens. After production, it can be stored in a stainless steel glass cabinet with lifting device[5].The choice of preservative drug is new and environmentally friendly. Although the existing products can not completely replace formalin, most commonly used are non-irritating scents and emitting a light Chinese herbal solution. For example, a polyhydroxymethyl heteroatom compound (10\% -30\%) is combined with a buffer solution (70\% $-90 \%$ and $\mathrm{pH}=4.3-6.5)$. The drug concentration can be checked once within 2 months.

\section{C.Human mummy specimens}

\section{a.production}

For the production of human mummy specimen, it is made by removing the internal organs and bone marrow after dissection. The concrete methods are as follows: first, the body is soaked in the anticorrosion solution, and the oil is dissolved and the skin is washed away. After 70 days the body was removed and dried. Fill the body cavity with spices and apply gum to the outside to prevent the body from touching the air and bacteria. Finally, the body is tightly wrapped up.

\section{b.preservation}

It needs to be anticorrosive for the preservation of human mummy specimens. For anticorrosion. The specific measures for corrosion: Injecting $0.1 \%-0.2 \%$ of 1059 injection for anti-corrosion and pest control by the use of 5-20ml drug injection. The injection sites include the trapezius, the latissimus dorsi and the thoracolumbar fascia.The injection amount was increased at the junction gap[6]. In addition, it can be brushed in glycerin once a month to replace once three months, so as to completely control mildew and meat insects. You can check it once a month to see if it is well preserved. 


\section{Human cast specimens}

\section{a. production}

Intubation is first carried out in the production of this kind of specimen.The method of direct ligation, thread ligation and suture ligation can be used. Secondly, perfusion is carried out. The color of the distal skin of the extremities should be observed before the first infusion, to see if there is a color of the filling agent. If the exudation is found, it can be supplemented. The plastic filling agent can be polyvinyl chloride, polystyrene, epoxy resin and so on. The alloy filler is usually made of lead, tin, cadmium and other alloys, which can be perfused with the inner ear, the bronchial tree and the ventricle. Third, corrosion. It is mainly to show the cast of the pipe. Acid corrosion, alkali corrosion, natural corrosion and carbonization can be used. For example, the sodium hypochlorite solution is used to corrode the washed specimen and then be immersed in weak acid without bleaching. Finally, flushing and refurbishing.Washing thoroughly with water[7]. If we want the bones to be white and beautiful, we soak it with hydrogen peroxide (5\%-10\%) solution for 24 hours. For the renovation, you can remove the clot, cut the branches, heat orthopedic and replant.

\section{b. preservation}

The wet preservation method is mainly used in the preservation of this kind of specimen, and the solution is made up by $5 \%$ formaldehyde and distilled water. The color of the specimen is brighter and glittering.

\section{E. Bio plastic specimens}

This kind of specimen needs to be plasticized while producing.The steps are storage, dissection, dehydration, slicing and vacuum replacement. In the storage, $20 \%$ formalin is required for the body to be perfused and soaked for at least 4 months. Dehydrate after the rotting tissue is removed[8].In the frozen state, the body was cut into $3.5 \mathrm{~mm}$ slices by using a saw. Design the posture after that. Finally, vacuum replacement is carried out. For example, Figure II is a vacuum-packed chest tomographic specimen.

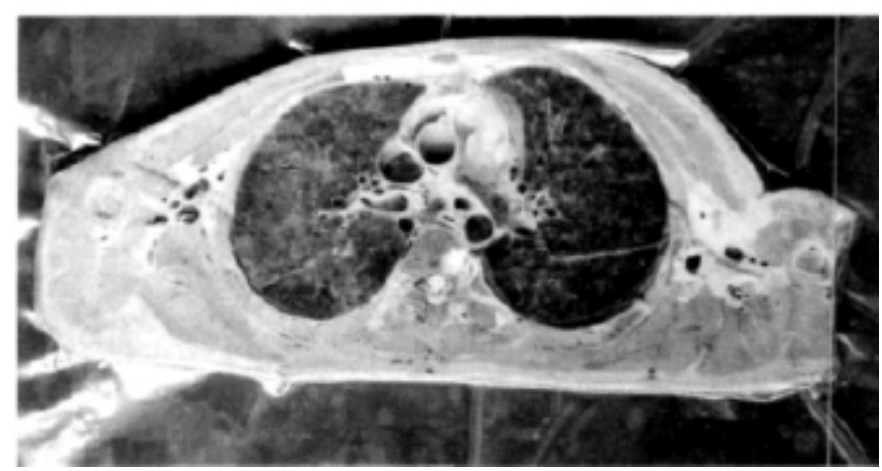

Figure II a vacuum-packed chest tomographic specimen.

This kind of specimen can be transformed into permanent preservation, which can be widely used in the field of teaching and research. It can completely replace the formalin soaked specimens in the future development, which is the development direction of the specimen production and preservation technology.

\section{Conclusion}

To sum up, through the technology and development of the production and preservation of the human anatomy specimen, We understand the connotation of human anatomy. We explain the current technology and development of human anatomy specimen preservation through ordinary human specimens, human mummy specimens, human cast specimens and bio plasticized specimens. To provide some reference for the researchers in the production and preservation of human anatomical specimens, and point out the development direction for the production of high quality human anatomical specimens in the future. 


\section{Reference}

[1]Ji Jingxiu,Su Daowen.Selection of Human Anatomical Specimen Preservation Dolution[J].Journal of Clinical Medicine Electronic,2016,3(27):5513-5514.

[2]Qiao Xing. Analysis of the methods and steps for the production of human anatomy plasticized specimens[J].Journal of Modern Health(Second Half Edition) 2016, (6): 279-279.DOI:10.3969/j.issn.1671-0223(b).2016.06.257.

[3]Gan Cheng,Tian Jia,Liu Liqiang.,etc.Study on Antiseptic and Fixation Techniques and Intravascular Lactose Perfusion Technique of Anatomical Specimens of Human Head and Facial[J].Journal of Chinese Aesthetic Medicine,2015,(12):44-49.

[4]Fang Jie,Yan Yanliang .Production and research progress of human anatomical special specimens[J].Jounalof Chinese Health Nutrition,2016,26(21):361-362.

[5]Zhou Zhangfu.Design and Comprehensive Utilization of Human Anatomical Research Specimens[J].Journal of Sichuan Anatomy, 2015, 23(2):60-62. DOI:10.3969/j.issn. 1005-1457.2015.044.

[6]Yi Zhiyong,Guo Xing.Application of Plasticized Specimen in Making Human Anatomical Specimens[J].Medical Information Journal,2013,(7):495-495.

[7]Huang Jun,Guo Xing,Guo Zhongxian .,etc.Application of Plasticized Specimen in Making Human Anatomical Specimens[J].Journal of Xiangnan University (Medical Science),2013,15(3):75-76.DOI:10.3969/j.issn.1673-498x.2013.03.030.

[8]Yin Hao,Zhu Lei,You Chao .,etc.Improvement of preservation method of neurosurgery microdissection specimens[J].Journal ofChinese Neurosurgery, 2011,27(9):969-970.DOI:10.3760/cma.j.issn.1001-2346.2011.09.034. 